\title{
The Study of Hydrodynamic Processes Occurring on Transition of Sudden Expanding of Hydraulic Section of Plane - Parallel Full Pipe Flow
}

\author{
Arestak Sarukhanyan ${ }^{1}$, Arevshad Vartanyan ${ }^{2}$, \\ Garnik Vermishyan ${ }^{1}$, Vache Tokmajyan ${ }^{3}$ \\ ${ }^{1}$ National University of Architecture and Construction of Armenia, Teryan Street, 105 \\ 375009, Yerevan, Republic of Armenia \\ ${ }^{2}$ Moscow Aviation Institute (National Research University), Volokolamskoe Highway, 4, \\ 125993, Moscow, Russia \\ ${ }^{3}$ Shushi University of Technology, Ashot Bekor, 4, 375000, Shushi
}

\begin{abstract}
The study regarding the regularity of changes in the hydrodynamic processes in the area of a sudden expanding in hydraulic section of the planeparallel full pipe flow of the liquid is carried out on the basis of the equations on the boundary layer. A method has been developed for determining the changes in the hydrodynamic parameters of the flow in the transition section which makes it possible to obtain the profile of the distribution of fluid velocities in any cross section of the channel based on the results of deformation of the areas of velocity under common initial and boundary conditions. The hydrodynamic processes occurring on transition of sudden expanding on hydraulic section of plane-parallel full pipe flow are studied in cases when: a) the velocity at any point of the inlet section of the channel is constant; b) the velocity at the inlet section is distributed according to the parabolic law. The calculation results for various values of the coefficient of expansion are given: $\alpha=\frac{a}{h}=0,3 ; 0,5 ; 0,7$.
\end{abstract}

DOI: 10.18421/TEM94-23

https://doi.org/10.18421/TEM94-23

Corresponding author: Arevshad Vartanyan, Moscow Aviation Institute (National Research University), Moscow, Russia.

Email: arevshadvartanyan@mail.ru

Received: 14 September 2020.

Revised: 03 November 2020.

Accepted: 11 November 2020.

Published: 27 November 2020.

(cc) BY-NC-ND (C) 2020 Arevshad Vartanyan et al; published by UIKTEN. This work is licensed under the Creative Commons Attribution-NonCommercial-NoDerivs 4.0 License.

The article is published with Open Access at www.temjournal.com
Based on the results of computer simulation, the course of deformation of the velocity diagram along the length of the transition section was obtained for a constant and parabolic distribution of the velocities of the fluid flowing into the expanded section of the channel. The regularities of pressure distribution along the length of the studied section were also determined.

Keywords - transition; hydraulic section; function; velocity; distribution.

\section{Introduction}

As a result of the analysis of studies on hydrodynamic processes occurring on transition of hydraulic section of full pipe flow, various theoretical and experimental methods have been developed. Each method of calculation is based on conditions of the structure and nature of the movement according to which theoretical studies are carried out and practical conclusions are made. Often these conclusions provide acceptable results for relatively small ranges of movement thus limiting their practical application. The study of the transition of the hydraulic section of a flat pipe using methods of boundary layer was first carried out in the work of Leybenzon [1]. However, the suggested method of calculation requires a bulk of calculations. In addition, the accuracy of calculation greatly reduces the hydraulic section which also limits its application.

The study of the transition of the inlet section of the plane-parallel full pipe flow by the method with regard to numerical integration of the system of equations of the boundary layer was carried out by $G$. Shlikhting [2]. Along with the numerical method, the author also developed an analytical method for calculating the transitional section [3] which is based on the condition for dividing the area of fluid motion 
in the pipe into two parts: the central section where the velocities are equal and the section near the fixed walls where the velocities vary along the regularities of the boundary layer. The developed method allows to obtain the distribution of the areas of fluid velocity in any hydraulic section of the channel transition.

The distributions of velocities in the transition of the inlet section of a circular cylindrical pipe were first obtained, and [4] suggested a numerical method for determining the deviation of velocities from their stated values. However, the results of his calculations give significant deviations, especially near the inlet section. Within the framework of the model of boundary layer [5], L. Schiller obtained the distribution of velocities in the transition of the inlet section of a circular cylindrical channel and developed a method for its calculation [6]. The obtained results show proper coincidence in the central part of the fluid movement in the pipe and significant deviations are observed near the fixed wall [7].

Investigations of the hydrodynamic parameters of the flow at the transition of the inlet section of cylindrical channels were also carried out by N.A. Slezkin [8]. Having approximated the Navier-Stokes equations for a viscous fluid, they formulated and solved a problem of boundary value for such types of channels, and the results were compared with experimental data. The analysis confirmed the reliability of the studies. However, these studies are unacceptable for cases where, depending on a sudden change in the geometric parameters of the channel, the deformation occurs in areas of velocity. Therefore, the determination of the regularities of changes in hydrodynamic parameters in these areas is of great theoretical and practical importance.

A similar study was carried out in [9], where approximate solutions of hydrodynamic parameters were obtained under conditions of isothermal laminar movement on the transition of the inlet section of flat and round cylindrical channels providing satisfactory results. Under these conditions studies were also carried out in three-dimensional space by the method of numerical integration of the Navier-Stokes equations [10] and the distributions of velocities and pressure in the transition section of the channel were determined. A comparative analysis of the results obtained by various methods of numerical integration was carried out [11]. Based on the condition of the square distribution of velocities in the area of boundary layer, the distributions of velocities and pressures in the transition section of the pipe were obtained [12].

A method has been developed [13] for cylindrical channels of arbitrary shape which enables to establish patterns of change in velocities and pressures on the transition of the inlet section of circular and flat channels. A comparative analysis was carried out and practical conclusions were drawn. In [14] within the framework of a twodimensional space the regularities of the change in velocities and pressures for the laminar movement of a liquid are determined. Let us note that in all the works mentioned above, the topics of study were the transitions of the inlet sections of channels of different cross-sections. In this case the results of the studies carried out are unacceptable for other transitional sections where due to a sudden change in the geometric parameters of the channels, the deformation of the areas of velocity occurs. For turbulent flows of a two-phase fluid, the dependences given in the work [15] can be used.

On the transition of sudden expanding of cross section of round pipe $(D / d=4)$ in case of laminar movement of viscous-plastic fluid the lines of electrical current are established by numerical equations, the changes of velocity and pressure along the channel are determined. In the work [16] expanded experimental studies were carried out in the area of sudden expanding of hydraulic section. Using magnetic-resonant tomographic method the qualitative estimations of the degree of changeability of the velocity were obtained. The qualitative analysis of stated equations of Navier-Stokes has been done in case of symmetric and asymmetric expansion of cross section of the pipe and the obtained non-linear system of differential equations were determined by numerical integration [17]. The results were compared with the experiments. On the sites of sudden expansion of cross section great experimental studies were carried out [18], [19]. The experimental setting was set out for this purpose and research was carried out in the areas of sudden expanding in case of $d / D=0,22 ; 0,5 ; 0,85$. The studies were carried out for Newtonian and non-Newtonian fluids [20].

\section{Conflict Setting}

In the transitional sections of the movement of a viscous fluid (inlet and outlet sections of the channel, sudden expansion and contraction of the hydraulic section etc.), a redistribution of the areas of velocity occurs which leads to a change in the hydrodynamic parameters of the fluid flow. The purpose of this study is to identify the regularities of changes in hydrodynamic parameters of a viscous fluid at any point of the hydraulic section of the channel and to develop a method for calculating energy losses.

\section{Theoretical Background}

In the work in the study of the transition of the inlet section of a round pipe at free initial velocity distributions for the laminar movement of a viscous fluid was carried out. Under these conditions we have asymmetric movement. In the inlet section of a round pipe, the inflowing liquid has a random 
distribution of velocity. When entering the pipe, the velocity of the liquid vanishes and the diagram of velocity deforms on the wall due to adhesion. A boundary layer forms near the fixed wall where the gradient of velocity becomes significant as a result of which the friction forces also obtain big values. The boundary layer gradually expands and encompasses the entire hydraulic section. The main factors for changing the hydrodynamic parameters of the liquid flow are its viscosity in the transitional section. This confirms the fact that the study of the regularities of changes in the hydrodynamic parameters in the transition areas has to be carried out according to the basic equations of the boundary layer.
Since viscosity forces mainly act in the boundary layer omitting all negligible numbers in the NavierStokes equation, we obtain a system of equations of boundary layer [3], [6]. Taking into account the above-mentioned considerations, theoretical and experimental studies of the transition of the inlet section of a round and flat pipe have been carried out.

\section{Methodology}

Let us consider the hydrodynamic processes occurring on the sites of sudden expansion of hydraulic section of flat pipe in case of laminar movement of viscous fluid (Figure 1).

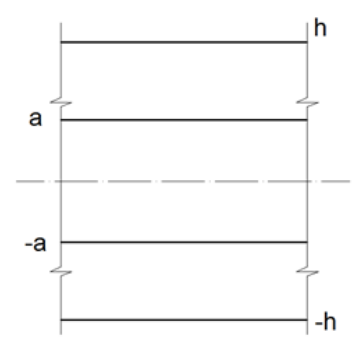

Figure 1. The scheme of sudden expanding of hydraulic section of flat pipe

As a result of sudden expansion of cross section of flat pipe the deformation of the areas of velocity of fluid movement occurs. The definition of hydrodynamic parameters of the flow in the area of redistribution of velocity in case of laminar movement of viscous liquid is stated by the methodology of the study of inlet section of cylinder channels [7], [8]. The system of equations of boundary layer in Cartesian coordinate system has the following form [3], [5]:

$$
\begin{aligned}
& V_{z} \frac{\partial V_{z}}{\partial z}+V_{y} \frac{\partial V_{z}}{\partial y}=-\frac{1}{\rho} \frac{\partial P}{\partial z}+v \frac{\partial^{2} V_{z}}{\partial y^{2}}, \\
& \frac{\partial V_{z}}{\partial z}+\frac{\partial V_{z}}{\partial y}=0 .
\end{aligned}
$$

For simplifying the equation (1) let us take omission from [2], [3], according to which $V_{y}=0$, we will get

$$
V_{z} \frac{\partial V_{z}}{\partial z}=-\frac{1}{\rho} \frac{\partial P}{\partial z}+v \frac{\partial^{2} V_{z}}{\partial y^{2}}
$$

For integration of received non linear differential equations we substitute the coefficient of the member $\frac{\partial V_{z}}{\partial z}$ of average speed, i.e. we take that $V_{z}=u_{0}$, where

$$
u_{0}=\frac{1}{2 h} \int_{-h}^{+h} \varphi(y) d y,
$$

Here $u=\varphi(y)$ - function of distributing the velocity of fluid inflowing into sudden expanding area.
Taking into account these omissions for hydrodynamic parameters of the flow on transition of sudden expansion of cross section of flat channel we have the following system of equations:

$$
\begin{aligned}
& u_{0} \frac{\partial V_{z}}{\partial z}=-\frac{1}{\rho} \frac{\partial P}{\partial z}+v \frac{\partial^{2} V_{z}}{\partial y^{2}}, \\
& \frac{\partial V_{z}}{\partial z}+\frac{\partial V_{z}}{\partial y}=0 .
\end{aligned}
$$

In case of following initial and boundary conditions we have:

$$
\begin{aligned}
& V_{z}=0, V_{y}=0 \text { in case } y=a z=0, \\
& V_{z}=\phi(y) \text { in case } z=0,-a \leq y \leq+a, \\
& V_{z} \rightarrow V^{\prime} \text { in case } z \rightarrow \infty,-h \leq y \leq+h .
\end{aligned}
$$

Here $V^{\prime}$ - is the velocity of the movement of the fluid on stabilized area determined by the following formula

$$
\frac{1}{\rho} \frac{\partial P}{\partial z}=v \frac{\partial^{2} V^{\prime}}{\partial y^{2}} .
$$

The solution of the equation (10) has the following expression [2]:

$$
V^{\prime}=-\frac{1}{\rho} \frac{\partial P}{\partial z} \frac{h^{2}}{2 v}\left(1-\frac{y^{2}}{h^{2}}\right)=\frac{3}{2} u_{0}\left(1-\frac{y^{2}}{h^{2}}\right),
$$

Where $u_{0}=\frac{2 V_{\max }}{\frac{h^{2} \partial p}{3 \rho v \partial x}} \quad u_{0}=\frac{2 V_{\max }^{\prime}}{3 \frac{h^{2} \partial P}{3 \nu \rho \partial z}}-$ is the average

velocity of hydraulic section. 
From the equation (5), (6) follows that in each fixed section of transition $\frac{\partial P}{\partial r}=0$. Consequently, the pressure depends only on the coordinates of $z$, i.e. $P=P(z)$.

The solution for the equation (5) with boundary conditions (7), (8), (9) is:

$$
V_{z}(z, y)=\sum_{k=1}^{\infty} C_{k}\left[\cos \left(\frac{\gamma_{k} y}{h}\right)-\frac{\sin \gamma_{k}}{\gamma_{k}}\right] \exp \left(-\frac{\gamma_{k}^{2} z}{h \cdot R e}() \frac{3 u_{0}}{2}\left(1-\frac{y^{2}}{h^{2}}\right)\right), \quad-h<y<+h .
$$

The specific meanings of the tasks are determined from boundary conditions: in case of $y= \pm h, V_{z}=0$ we will get the equation $\cos \left(\frac{\gamma_{k} y}{h}\right)-\frac{\sin \gamma_{k}}{\gamma_{k}}=0$ or

$\operatorname{tg} \gamma_{k}=\gamma_{k}$, for finding specific meanings. For determining the meanings of coefficients $C_{k}$ we use boundary conditions (8). As a result, we get

$$
\phi(y)=\sum_{k=1}^{\infty} C_{k}\left[\cos \left(\frac{\gamma_{k} y}{h}\right)-\frac{\sin \gamma_{k}}{\gamma_{k}}\right]+\frac{3 u_{0}}{2}\left(1-\frac{y^{2}}{h^{2}}\right), \quad-a<y<+a .
$$

Multiplying both parts of the equation (13) by corresponding orthogonal functions

$$
\Phi_{k}\left(\gamma_{k} \frac{y}{h}\right)=\frac{1}{2 h \sin ^{2} \gamma_{k}}\left[\cos \left(\frac{\gamma_{k} y}{h}\right)-\frac{\sin \gamma_{k}}{\gamma_{k}}=0\right]
$$

And by integrating in the interval $-h \leq y<+h$, we get the meaning for coefficient $C_{k}$ :

$$
C_{k}=\frac{1}{2 h \sin ^{2} \gamma_{k}} \int_{-h}^{h}\left[\varphi(y)-\frac{3 u_{0}}{2}\left(1-\frac{y^{2}}{h^{2}}\right)\right]\left[\cos \left(\frac{\gamma_{k} y}{h}\right)-\cos \gamma_{k}\right] d y
$$

Let us express the relation (15) in the form of

Where

$$
C_{k}=\frac{1}{\sin ^{2} \gamma_{k}}\left[L_{1}+2 u_{0} h \cos \gamma_{k}\right]
$$

$$
L_{1}=\int_{-a}^{a} \frac{\varphi(y)}{h}\left[\cos \left(\frac{\gamma_{k} y}{h}\right)-\cos \gamma_{k}\right] d y \text {. }
$$

\section{Results}

The distribution of the pressure for studied part of the pipe is determined by system of equations (5), (12):

$$
-\frac{1}{\rho} \cdot \frac{\partial P}{\partial z}=\sum_{k=1}^{\infty} C_{k}\left[\left(\frac{v \gamma_{k}^{2}}{h^{2}}-\frac{u_{0} \gamma_{k}^{2}}{h \operatorname{Re}}\right) \cos \left(\frac{\gamma_{k} y}{h}\right)-u_{0} \cos \gamma_{k}\right] \exp \left(-\frac{\gamma_{k}^{2} z}{h \cdot \operatorname{Re}}\right)+\frac{3 u_{0} v}{h^{2}}
$$

From the equation (18) it follows that in case of $z \rightarrow \infty$, the regularity of the change of the pressure coincides with the regularity of the change in the case of current laminar movement, i.e.

$$
\left(-\frac{\partial P}{\partial z}\right)_{z \rightarrow \infty}=\frac{3 u_{0} v}{h^{2}}
$$

Integrating the equation (18) we get distribution of the pressure along the length

$$
\frac{P_{0}-P}{\rho}=\sum_{k=1}^{\infty} C_{k}\left[\left(u_{0}-\frac{v \operatorname{Re}}{h}\right) \cos \left(\frac{\gamma_{k} y}{h}\right)+\frac{u_{0} h \operatorname{Re}}{\gamma_{k}^{2}} \cos \gamma_{k}\right] \exp \left(-\frac{\gamma_{k}^{2} z}{h \cdot \operatorname{Re}}\right)+\frac{3 u_{0} v}{h^{2}} z .
$$

Let us note that from equation (20) it follows: $P_{0}=P$ if $z=0$.

The solutions are obtained for both boundary and initial conditions. Let us study two private cases of solving the tasks. 


\section{Discussion}

Case I. Suppose that the velocity of fluid flowing into suddenly expanding section in all parts of hydraulic section is constant, consequently we have

$\varphi(r)=u_{0}^{*}=$ const,$\quad-a<y<+a$. Let us distinguish the meaning of the integral $L_{1}$ for this case:

$$
L_{1}=\int_{0}^{a} u_{0}^{*}\left[\cos \left(\frac{\gamma_{k} y}{h}\right)-\cos \gamma_{k}\right] d y=2 u_{0}^{*} a\left[\frac{\sin \gamma_{k} \frac{a}{h}}{\gamma_{k} \frac{a}{h}}-\cos \gamma_{k}\right]
$$

Using the value $L_{1}$ from (21) in (16) and taking into account that $u_{0}=\frac{u_{0}^{*} a}{h}$, we get the following for coefficients $C_{k}$ :

$$
\begin{aligned}
C_{k}=\frac{u_{0}}{\alpha \gamma_{k} \sin ^{2} \gamma_{k}} & \sin \frac{\gamma_{k} \alpha}{h} . \\
\bar{u} & =\sum_{k=1}^{\infty} \frac{\sin \alpha \gamma_{k}}{\alpha \gamma_{k} \sin ^{2} \gamma_{k}}\left[\cos \left(\gamma_{k} \bar{y}\right)-\cos \gamma_{k}\right] \exp \left(-\gamma_{k}^{2} \bar{z}\right)+\frac{3}{2}\left(1-\bar{y}^{2}\right) .
\end{aligned}
$$

Having the value for coefficients $C_{k}$, we get distribution of velocity on transition of sudden expansion of cross section of flat channel in dimensionless form:
Here dimensionless values are pointed in the form of $\bar{u}=\frac{V_{z}}{u_{0}}, \alpha=\frac{a}{h}, \bar{y}=\frac{y}{h}, \bar{z}=\frac{z}{h \cdot R e}$.

In Figure 2, Figure 3, Figure 4 the numerical calculations are given for the cases $\alpha=0,3 ; 0,5 ; 0,7$. for sudden expansion of cross section of flat channel in conditions of constant velocity of fluid inflowing to expanded section. In Figure 2 the graph of velocity in transition part is given, and in Figure 3 we have their distribution along the transition part in case of $\alpha=0,7$. In the Figure 4 we have the graph of velocity $\left(\frac{u}{u_{0}}=f(z)\right)$ in the parts of hydraulic section $\bar{y}=\frac{y}{h}=0 ; 0,2 ; 0,4 ; 0,6 ; 0,8 ; 0,9$ in case of $\alpha=0,5$. It follows from all the graphs that the velocities having constant values are deformed and redistribution of velocities occur along the transition. In the end of transition, the regularity of changes of velocities becomes parabolic.

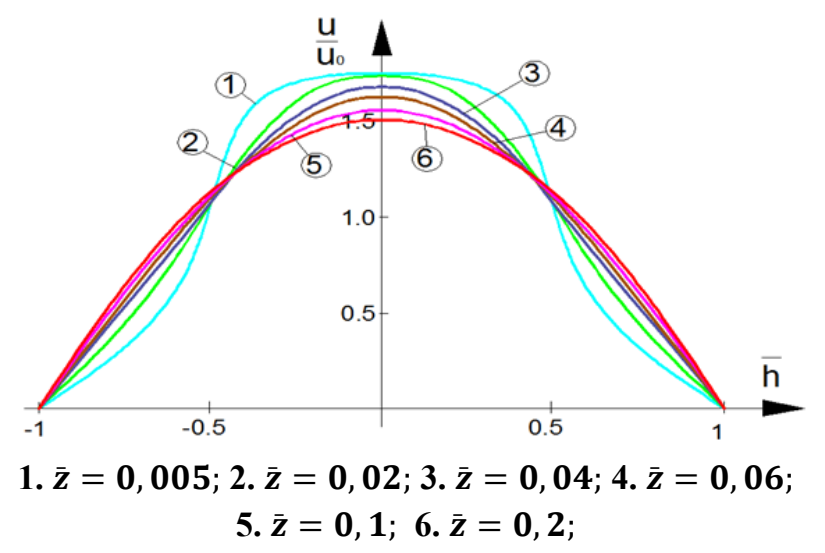

Figure 2. Joint graph of velocity change on transitional section

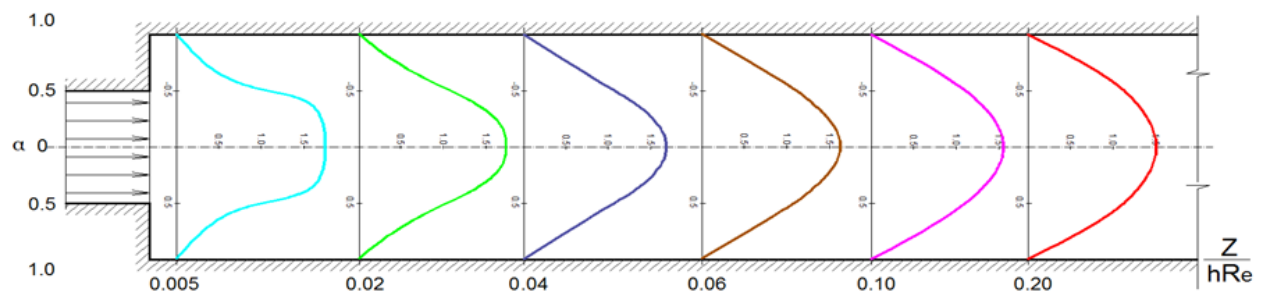

Figure 3. Graphs of velocity change along the transitional section depending on the coordinates $\bar{z}$ and $\bar{y}$ 


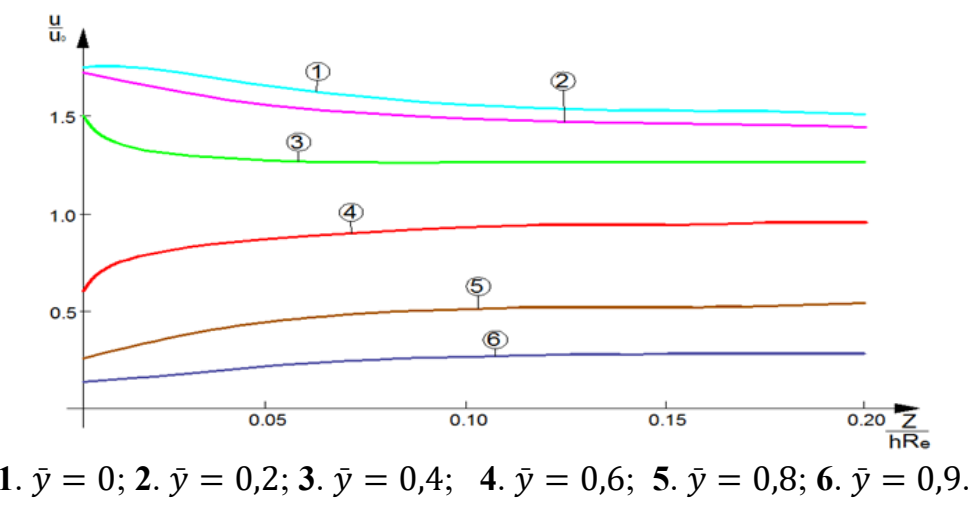

Figure 4. Graphs of velocity change in the parts of hydraulic section $\bar{y}=0 ; 0,4 ; 0,8 ; 0,9$ along the length of transition

\section{area}

From the given graphs we get the length of transitional area $\bar{z}=0,186$ and taking into account the condition that the velocity on the axis of flow in case of $\bar{y}=0$ is equal to $\frac{u}{u_{0}}=0,99$ we get the length Inserting the value of the coefficient $C_{k}$ from (22) into the equation (20), we get the distribution of pressure along the length of transition area of sudden expanding of cross section of flat channel: of transitional section.

$$
\frac{P_{0}-P}{\rho}=\sum_{k=1}^{\infty} \frac{u_{0} h}{a \gamma_{k} \sin ^{2} \gamma_{k}} \sin \frac{\gamma_{k} a}{h}\left[\left(u_{0}-\frac{v R e}{h}() \cos \frac{\gamma_{k} y}{h} \frac{u_{0} h R e}{\gamma_{k}^{2} \cos \gamma_{k}}\right) \exp \left(-\frac{\gamma_{k}^{2} z}{h R e}() \frac{3 u_{0} v}{h^{2}}\right)\right]
$$

Case II. Let us think that the distribution of velocity of fluid on the area of sudden expansion is parabolic. In this case we have: $\varphi(y)=\frac{3}{2}$.

$$
L_{1}=\frac{3}{2} \frac{u_{0}}{\alpha} \int_{-a}^{a}\left(1-\frac{y^{2}}{a^{2}}\right)\left[\cos \left(\frac{\gamma_{k} y}{h}\right)-\cos \gamma_{k}\right] d y=3 u_{0} h\left[\frac{2}{\alpha^{3} \gamma_{k}^{3}}\left(\sin \alpha \gamma_{k}-\alpha \gamma_{k} \cos \gamma_{k}\right)\right]-2 u_{0} h \cos \gamma_{k}
$$

Inserting the value of integral $L_{1}$ into the equation (16) we will get the following for coefficient $C_{k}$ :

$$
C_{k}=\frac{3 u_{0}}{\alpha^{3} \gamma_{k}^{3} \sin ^{2} \gamma_{k}}\left(\sin \alpha \gamma_{k}-\alpha \gamma_{k} \cos \alpha \gamma_{k}\right)
$$

$\frac{u_{0}}{\alpha}\left(1-\frac{y^{2}}{h^{2}}\right),-a \leq y<+a$. Then the value of the integral $L_{1}$ will be:

$$
\bar{u}=\frac{3}{2}(1-\bar{y})+\frac{3}{\alpha^{3}} \sum_{k=1}^{\infty} \frac{1}{\gamma_{k}^{3} \sin ^{2} \gamma_{k}}\left(\sin \alpha \gamma_{k}-\alpha \gamma_{k} \cos \alpha \gamma_{k}\right)\left(\cos \gamma_{k} \bar{y}-\cos \gamma_{k}\right) \exp \left(-\gamma_{k}^{2} \bar{z}\right) .
$$

For the purpose of obtaining graphical figure of regulation of the changes of velocity on transition area of sudden expansion of cross section of flat channel in case of parabolic distribution of fluid velocity inflowing to suddenly expanded area, the
Having the value of coefficient $C_{k}$ from the equation (12), we will get the distribution of velocities on transitional area of sudden expansion of cross section of flat dimensionless channel:

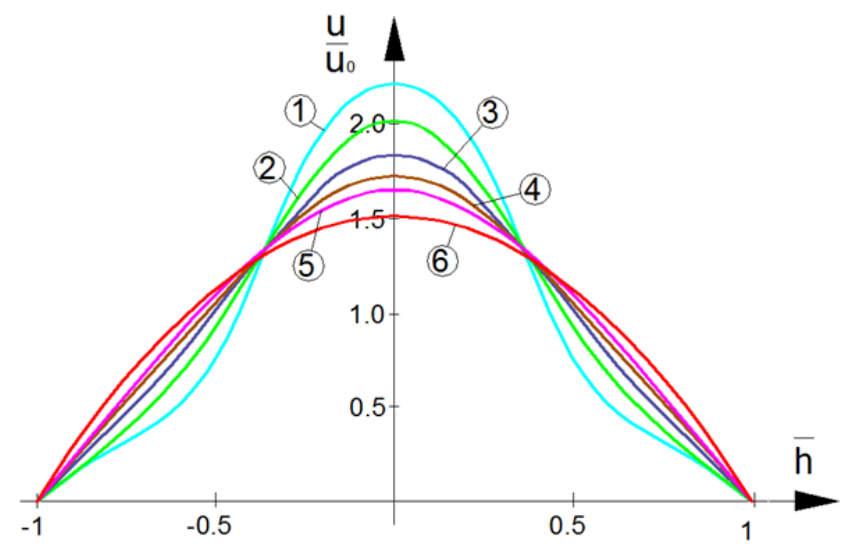

1. $\bar{z}=0,005$; 2. $\bar{z}=0,02$; 3. $\bar{z}=0,04 ;$ 4. $\bar{z}=0,06$; 5. $\bar{z}=0,08$; 6. $\bar{z}=0,2$.

Figure 5. Graph of change of velocity on transitional area 
In Fig. 7 the graphs of velocity $\left(\frac{u}{u_{0}}=f(\bar{z})\right)$ in the parts of hydraulic section are given $\bar{y}=\frac{y}{h}=$ $0 ; 0,2 ; 0,4 ; 0,6 ; 0,8 ; 0,9$ when $\alpha=0,5$. We see from these that the velocity of the fluid inflowing to suddenly expanding area from parabolic value $u=2 u_{0}^{*}\left(1-\frac{y^{2}}{a^{2}}\right)$ deforms and redistribution of velocities along the transitional area occurs. In the end of transitional area the distribution of velocities becomes parabolic.

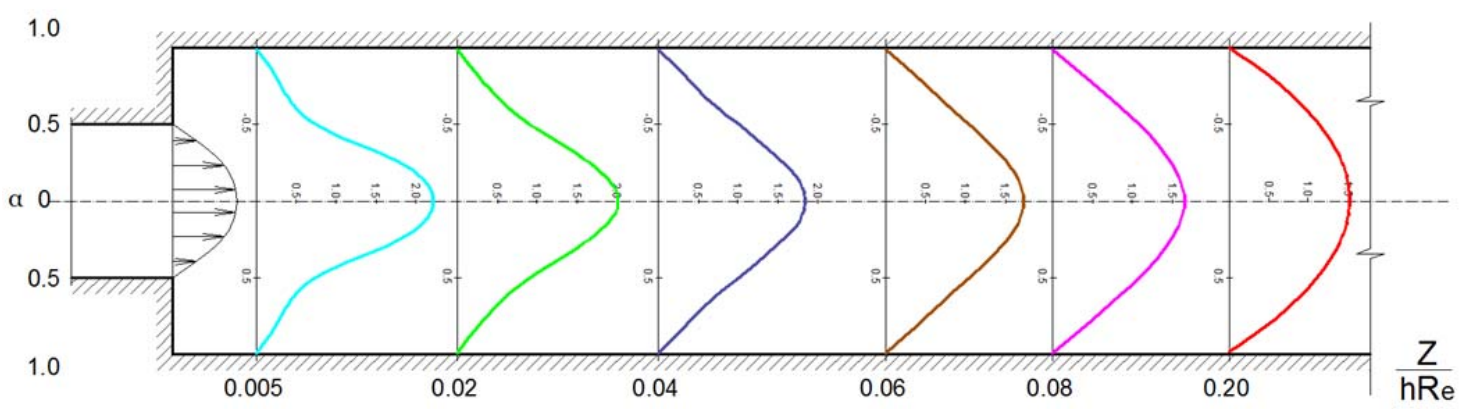

Figure 6. Graphs of changes of velocity along the length of transitional area depending on the coordinates: $\bar{z}$ and $\bar{y}$

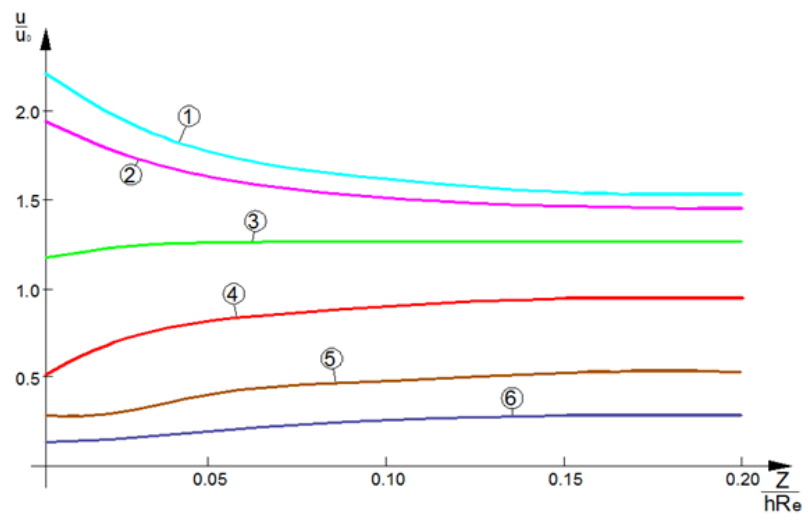

1. $\bar{y}=0 ; 2 . \bar{y}=0,2 ; 3 . \bar{y}=0,4 ; 4 . \bar{y}=0,6 ; 5 . \bar{y}=0,8 ; 6 . \bar{y}=0,9$

Figure 7. The graph of velocity change in the parts of hydraulic section $\bar{y}=0 ; 0,4 ; 0,8 ; 0,9$. along the length of transitional area

We see from the graphs that if the flow on axis $(\bar{y}=0): \frac{u}{u_{0}}=0,99$, then the length of transitional area is $\bar{z}=0.163$. Inserting the values of the coefficients $C_{k}$ from (25) related to (20), we will get the regularity of distribution of pressure along the transitional area:

$$
\begin{aligned}
& \frac{P_{0}-P}{\rho}=\sum_{k=1}^{\infty} \frac{u_{0}}{\sin ^{2} \gamma_{k}}\left[\frac{3 \sin \alpha \gamma_{k}}{\alpha^{3} \gamma_{k}^{3}}-\frac{3 \cos \alpha \gamma_{k}}{\alpha^{2} \gamma_{k}^{2}}\right]\left[\left(u_{0}-\frac{v \mathrm{Re}}{h}\right) \cos \left(\frac{\gamma_{k} y}{h}\right)+\frac{u_{0} h \mathrm{Re}}{\gamma_{k}^{2}} \cos \gamma_{k}\right] \exp \left(-\frac{\gamma_{k}^{2} z}{h \cdot \mathrm{Re}}\right)+ \\
& +\frac{3 u_{0} v}{h^{2}} z
\end{aligned}
$$




\section{Conclusions}

The suggested method for calculating the distribution of velocities in the transitional area of the sudden expansion of hydraulic section of a flat channel allows to obtain the distribution of velocities and pressure along the length under general initial and boundary conditions. From the obtained general solutions, the profiles of deformation of the velocity areas of the hydraulic section along the length of the transition are calculated in case of constant and parabolic distribution of the inflowing fluid to the expanded section. The distributions of velocities and pressure along the length of the transition section as well as its length have been determined.

\section{References}

[1]. Leybenzon, A. S. (1947). Movement of natural liquids and gases in porous media. Moscow, OGIZ.

[2]. Schlichting, H. (1934). Laminare kanaleinlaufstromung. Zamm, 14, 368-373.

[3]. Schlichting, H. (1968). Boundary layer theory (Vol. 960). New York: McGraw-Hill.

[4]. Boussinesq, J. (1903). Comptes Rendus de l'Àcad. des Sciences* T. 132, 1901. Théorie analytique de la chaleur, 2, 172.

[5]. Loytsyanskiy, L. G. (1973). Fluid and Gas Mechanics. Nauka, Moscow.

[6]. Schiller, L. (1936). The Motion of Liquid in Pipes [Russian translation]. Gostikhizdat, Moscow.

[7]. Targ, S. M. (1951). Main tasks of the theory of laminar flows. Publishing house of technical and theoretical literature.

[8]. Slezkin, N. A. (1955). Dynamics of a viscous incompressible fluid. Gostekhizdat, Moscow, 221-223.

[9]. Chen, R. (1973). Flow in the Entrance Region at Low Reynolds Numbers. J. Fluids, Eng., 95, 153-158.
[10]. Young, F. J. (2016). The Entrance Region of Circular Pipes Revisited. Open Access Library Journal, 3(07), 1.

[11]. Hornbeck, R. W. (1964). Laminar flow in the entrance region of a pipe. Applied Scientific Research, Section A, 13(1), 224-232.

[12]. Mohanty, A. K., \& Asthana, S. B. L. (1979). Laminar flow in the entrance region of a smooth pipe. Journal of Fluid Mechanics, 90(3), 433-447.

[13]. Sparrow, E. M., Lin, S. H., \& Lundgren, T. S. (1964). Flow development in the hydrodynamic entrance region of tubes and ducts. The Physics of Fluids, 7(3), 338-347.

[14]. Belyaev, L. A., Zaitsev, A. S., Kondakov, A. A., Shevelev, S. A., Valkov, E. P., \& Matveeva, A. A. (2015). Numerical analysis of fluid particles motion in curved ducts. In MATEC Web of Conferences. Vol. 37: Smart Grids 2015.—Les Ulis, 2015. (Vol. 372015, p. 1007).

[15]. Tokmajyan, H. V. (2016). On Movement of Suspended Particles in Turbulent Flow. Bulletin of High Technology, Shushi, (1), 3.

[16]. Mullin, T., Seddon, J. R. T., Mantle, M. D., \& Sederman, A. J. (2009). Bifurcation phenomena in the flow through a sudden expansion in a circular pipe. Physics of fluids, 21(1), 014110.

[17]. Hawa, T., \& Rusak, Z. (2000). Viscous flow in a slightly asymmetric channel with a sudden expansion. Physics of Fluids, 12(9), 2257-2267.

[18]. Gücüyen, E., Erdem, R. T., \& Gökkuş, Ü. (2019). Numerical Modelling Of Sudden Contraction In Pipe Flow. Sigma: Journal of Engineering \& Natural Sciences/Mühendislik ve Fen Bilimleri Dergisi.

[19]. Fester, V., Mbiya, B., \& Slatter, P. (2008). Energy losses of non-Newtonian fluids in sudden pipe contractions. Chemical Engineering Journal, 145(1), 57-63.

[20]. Durst, F., Ray, S., Ünsal, B., \& Bayoumi, O. (2005). The development lengths of laminar pipe and channel flows. Journal of fluids engineering, 127(6), 11541160 . 